Revue de droit comparé du travail et de la sécurité sociale

$3 \mid 2019$

Les migrations internationales de travail

\title{
La réforme du travail à temps partiel
}

Achim Seifert

\section{OpenEdition}

Journals

Édition électronique

URL : https://journals.openedition.org/rdctss/1517

ISSN : 2262-9815

Éditeur

Centre de droit comparé du travail et de la sécurité sociale

Édition imprimée

Date de publication : 1 novembre 2019

Pagination : 188-191

ISSN : 2117-4350

Référence électronique

Achim Seifert, « La réforme du travail à temps partiel », Revue de droit comparé du travail et de la sécurité sociale [En ligne], 3| 2019, mis en ligne le 01 novembre 2021, consulté le 12 novembre 2021. URL : http://journals.openedition.org/rdctss/1517 Licence Creative Commons Attribution - Pas d'Utilisation Commerciale - Pas de Modification 4.0 International. 


\section{ACHIM SEIFERT}

UNIVERSITÉ FRIEDRICH SCHILLER DE IÉNA

\section{LA RÉFORME DU TRAVAIL À TEMPS PARTIEL}

En 2019, I'actualité juridique du droit du travail en Allemagne a principalement

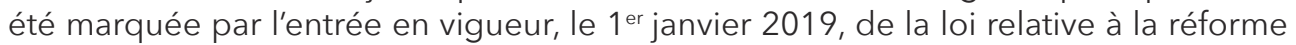
du droit du travail à temps partiel ${ }^{1}$. Pour l'essentiel, cette réforme introduit un droit au travail à temps partiel pour une durée déterminée (I) et modifie le régime du travail sur appel (II).

\section{I - LE DROIT AU TRAVAIL À TEMPS PARTIEL POUR UNE DURÉE DÉTERMINÉE}

Depuis l'adoption de la loi relative au temps partiel et au travail à durée déterminée (Teilzeit- und Befristungsgesetz TzBfG) du 21 décembre 2000, le droit allemand reconnaît de manière générale que, dans les entreprises de 15 salariés ou plus, le salarié dispose du droit de demander à son employeur la réduction de sa durée du travail, à moins que la réduction sollicitée n'entrave le bon fonctionnement de l'entreprise (article 8 TzBfG). De ce fait, le salarié a donc droit à la modification de son contrat de travail. En consacrant ce « droit au travail à temps partiel », le législateur fédéral poursuit son objectif de faciliter la conciliation entre vie professionnelle et vie privée, notamment pour les femmes salariées.

Toutefois, il a rapidement été mis en évidence que le passage au travail à temps partiel enferme souvent les salariées dans un "piège" dont elles ne peuvent que difficilement sortir: " une fois travail à temps partiel, toujours travail à temps partiel ». En effet, la loi ne prévoit pas un droit général du salarié qui a réduit sa durée du travail en vertu de l'article $8 \mathrm{TzBfG}$ à réclamer à son employeur le retour au statu quo ante, c'est-à-dire aux heures de travail précédant la réduction de la durée de travail. L'article 9 TzBfG engage seulement l'employeur à prendre en considération la demande formulée par un salarié à temps partiel qui souhaite retrouver son ancien

1 Loi sur le développement du droit à temps partiel - Introduction d'un temps partiel à durée déterminée (Gesetz zur Weiterentwicklung des Teilzeitrechts - Einführung einer Brückenteilzeit) du 11 décembre 2018, Journal Officiel de la RFA, 2018, Partie I, p. 2384. Pour une analyse plus détaillée de cette nouvelle loi, voir notamment F. Bayreuther, «Die neue Brückenteilzeit und andere Änderungen im TzBfG », Neue Zeitschrift für Arbeitsrecht (NZA), 2018, p. 1577; voir aussi l'exposé des motifs du législateur fédéral: Projet de loi du gouvernement fédéral pour le développement du droit à temps partiel - Introduction d'un temps partiel à durée déterminée, op. cit., BundestagsDrucksachen, 19/3452. 
temps de travail, et à lui donner priorité pour toute vacance d'un poste assimilé si les autres candidats sont à compétences égales. Un salarié à temps partiel est donc dépendant des fluctuations au sein de l'entreprise.

Ce n'est que dans certaines situations particulières que la loi prévoit implicitement un droit à une réduction du temps de travail pour une période déterminée. Ainsi, l'article 15 alinéas 5 et 7 de la loi relative à l'allocation parentale et au congé parental (Bundeselterngeld- und Elternzeitgesetz BEEG) cite notamment les cas du salarié en congé parental et du salarié qui réduit son temps de travail en vertu de l'article 3 alinéas $1^{\text {er }}$ et 5 de la loi relative au congé de solidarité familiale (Pflegezeitgesetz PflegeZG) du 28 mai 2008. En outre, plusieurs conventions collectives du travail entérinent un droit au travail partiel pour une période déterminée, à l'instar de l'article 11 de la Convention collective du secteur public (Tarifvertrag für den öffentlichen Dienst TVöD) et de la Convention collective de la métallurgie (2018).

La nouvelle loi relative à la réforme du droit du travail à temps partiel du 11 décembre 2018 modifie l'article 9 TzBfG en faisant peser sur l'employeur la charge de prouver que le salarié demandant le retour à son ancienne durée de travail n'est pas suffisamment apte à occuper un poste vacant par rapport à d'autres candidats. Cette modification, à première vue marginale, renforcera sans doute la position du salarié à temps partiel qui sollicite une extension de sa durée de travail.

Au cœur de cette réforme figure le nouvel article 9a TzBfG qui confère au salarié - sous certaines conditions légales - le droit à un travail à temps partiel pour une période déterminée (Brückenteilzeit). Dès lors, l'ancienne durée du travail sera automatiquement rétablie après l'expiration de la période déterminée par les parties. Cette nouvelle disposition de la TzBfG, issue de l'accord de coalition entre les partis chrétien-démocrate (CDU/CSU) et social-démocrate (SPD) et vivement réclamée par les sociaux-démocrates, tente d'apporter une réponse au "piège » qui peut résulter du travail à temps partiel.

S'agissant du champ d'application du droit à une réduction de la durée temporaire de travail, l'article 9a alinéa $1^{\text {er }}$ TzBfG exige que la relation de travail concernée soit continue depuis au moins 6 mois et que l'entreprise de l'employeur emploie plus de 45 salariés, apprentis non compris. En raison de ce seuil d'effectifs très élevé, la portée de ce nouveau droit restera nécessairement restreinte: en effet, une majorité de travailleurs est employée dans des entreprises de moins de 45 salariés.

Le salarié devra demander à l'employeur la réduction de sa durée de travail au plus tard trois mois avant le début souhaité. La loi n'exige pas que la demande du salarié soit motivée (par exemple par le besoin d'une garde d'enfants ou de soins à domicile d'un membre de la famille). L'employeur est tenu de discuter cette demande avec le salarié en vue d'un accord et de lui communiquer sa décision, au plus tard un mois avant le début de la réduction de la durée de travail.

Dans l'hypothèse où l'employeur ne rejette pas par écrit la demande du salarié au plus tard un mois avant le début de la réduction du temps de travail demandée par le salarié, l'employeur est présumé avoir accepté la demande (voir l'article 9a, alinéa 3, lu en combinaison avec l'article 8, alinéa 5 TzBfG). Par conséquent, le temps de travail 
sera automatiquement modifié en fonction des souhaits formulés par le salarié dans sa demande de réduction de la durée de travail.

L'employeur ne peut refuser une telle demande que dans la mesure où la réduction du temps de travail demandée entraverait le bon fonctionnement de l'entreprise (betriebliche Gründe). A cet égard, l'article 9a, alinéa 2 renvoie à l'article 8, alinéa 4 TzBfG qui précise les caractéristiques du bon fonctionnement de l'entreprise susceptibles d'être invoqués par l'employeur pour justifier son refus à un salarié. Ainsi, l'employeur pourra notamment arguer que la réduction temporaire de la durée de travail sollicitée par un salarié est susceptible d'affecter considérablement l'organisation du travail, la sécurité au travail, ou d'engendrer des coûts disproportionnés.

Pour éviter que l'employeur ne subisse une surcharge d'activité inhérente au droit à une réduction temporaire de la durée du travail, l'article 9a alinéa 2 TzBfG limite le nombre de salariés pouvant faire recours au droit à une réduction temporaire de la durée du travail dans les entreprises de 45 à 200 salariés. A titre d'exemple, l'employeur sera autorisé à refuser la demande du salarié lorsque, dans une entreprise de plus de 45 et moins de 60 salariés, déjà quatre salariés au moins bénéficient de cette mesure.

Après le retour du salarié à son ancien temps de travail, celui-ci ne pourra demander une nouvelle réduction de son temps de travail qu'après un délai de carence d'un an. Dans l'hypothèse où l'employeur a légitimement refusé une demande en vertu de l'article 9a TzBfG, le salarié ne pourra solliciter une autre demande de réduction temporaire de sa durée de travail qu'après deux ans.

\section{II - MODIFICATION DU RÉGIME DU TRAVAIL SUR APPEL}

Parmi les autres réformes introduites par la nouvelle loi, les dispositions concernant le travail sur appel méritent également une attention particulière.

L'ancien article 12, alinéa 1 er TzBfG obligeait l'employeur et le salarié à insérer dans leurcontratdetravailsurappeluneclause déterminantuneduréeminimalehebdomadaire et journalière de travail. Dans I'hypothèse où les parties ne fixaient pas la durée minimale hebdomadaire de travail, une durée de 10 heures était alors considérée comme convenue.

La nouvelle version de l'article 12, alinéa $1^{\text {er }}$ TzBfG, en vigueur depuis le $1^{\text {er janvier }}$ 2019, majore la durée hebdomadaire minimale de travail de 10 à 20 heures. Cette augmentation des heures de travail pourra avoir comme conséquence qu'un grand nombre de relations de travail sur appel jusqu'alors exemptées de la sécurité sociale - il s'agit des relations de travail dites insignifiantes (geringfügige Beschäftigungsverhältnisse) au sens de l'article 8 , alinéa $1^{\text {er }} n^{\circ} 2$ du Code de la sécurité sociale Partie IV (Sozialgesetzbuch SGB) -, seront désormais soumises au régime de la sécurité sociale.

Par ailleurs, le législateur fédéral a introduit, dans le nouvel article 12, alinéa 2 TzBfG, une disposition qui limite le pouvoir de l'employeur à recourir à la main-d'œuvre du salarié sur appel. 


\section{Allemagne}

Dans le cas où les parties ont fixé la durée hebdomadaire minimale du travail par convention, l'employeur ne pourra dépasser cette durée qu'à hauteur de $25 \%$. Si les parties ont convenu d'une durée hebdomadaire maximale du travail, celle-ci ne pourra être diminuée qu'à hauteur de 20 \% en dessous de ce seuil. Cette nouvelle disposition légale reprend la jurisprudence de la Cour fédérale du travail (Bundesarbeitsgericht - $B A G)^{2}$.

2 Cour fédérale du travail (BAG), arrêt du 24 septembre 2014, n 5 AZR 1024/12, NZA, 2014, p. 1328. 